\title{
High magnitude, in vitro, biaxial, cyclic tensile strain induces actin depolymerization in tendon cells
}

\author{
Michael Lavagnino \\ Keri L. Gardner \\ Steven P. Arnoczky
}

Laboratory for Comparative Orthopaedic Research, Michigan State University, USA

Corresponding author:

Michael Lavagnino

Laboratory for Comparative Orthopaedic Research, Michigan State University

784 Wilson Rd

48824 East Lansing, Michigan, USA

E-mail: lavagnin@cvm.msu.edu

\section{Summary}

Background: the cytoskeleton is a dynamic arrangement of actin filaments that maintain cell shape and are vital in mediating the mechanobiological response of the cell.

Methods: to determine the cytoskeletal response to varying in vitro, biaxial stretch amplitudes, rattail tendon cells were paired into control and cyclically strained groups of $4.75,9.5$, or $12 \%$ strain at $1 \mathrm{~Hz}$ for 2 hours and the actin cytoskeleton stained. The cells were analyzed for actin staining intensity as a measure of relative depolymerization and for cell shape. Collagenase gene expression was measured in cells undergoing $12 \%$ cyclic strain at $1 \mathrm{~Hz}$ for 24 hours.

Results: there was no significant difference in the degree of actin staining intensity between the control group and cells strained at either 4.75 or $9.5 \%$. However, cells strained at $12 \%$ demonstrated a significant decrease in actin staining intensity (depolymerization) compared to control cells, increased collagenase expression by $81 \%$, and a clear shift towards a more rounded cell shape.

Conclusion: the results of this study demonstrate that the previously reported induction of collagenase activity associated with the application of high magnitude, in vitro, tensile strains may actually be a result of cytoskeletal depolymerization, which causes loss of tensional homeostasis and alteration of cell shape.

KEY WORDS: actin intensity, cell shape, collagenase, mechanobiology, tendinopathy, under-stimulation.

\section{Introduction}

The ability of cells to respond to mechanical loads is central to the concept of mechanotransduction and the maintenance of tissue homeostasis ${ }^{1}$. In tendons and ligaments, the mechanobiological response of the cell is mediated, in large part, through the cytoskeleton, and alterations in the shape and structure of the cytoskeleton are known to influence a variety of changes in gene expression ${ }^{2}$.

While the precise level of mechanobiological stimulation required to maintain tendon homeostasis is not currently known, it is very likely that an abnormal level of stimulation may have a role in the etiopathogenesis of tendinopathy ${ }^{3,4}$. Numerous investigators have suggested that over-stimulation of tendon cells, secondary to repetitive loading, results in a pattern of gene expression that can lead to tendinopathy ${ }^{5-8}$. The presence of increased levels of interstitial collagenase mRNA and protein in ligament and tendon cells is a well-known marker of tendinopathy ${ }^{9}$. Physiologically high (15\%) strain magnitudes have been shown to up-regulate collagenase activity in ligament cells in monolayer culture $^{6}$. In contrast, numerous studies have also shown that the loss of cytoskeletal tensional homeostasis, secondary to stress deprivation of tendon cells in situ (under-stimulation), can markedly stimulate interstitial collagenase mRNA expression and protein synthesis ${ }^{10-14}$. The reasons for these conflicting observations remain unclear. Several studies have shown that chemically or physically induced disruptions of the actin cytoskeleton can trigger an up-regulation of collagenase (mRNA) in fibroblasts ${ }^{2,11-13,15}$. Loss of matrix stress through the release of cell-seeded collagen gels has been shown to cause the loss of cytoskeletal organization and an up-regulation of collagenase gene expression ${ }^{12,15}$. Likewise, an increase in actin depolymerization has been demonstrated in chondrocyte/agarose constructs exposed to $15 \%$ compressive strains ${ }^{16}$. Therefore, it is possible that tendon cells that are exposed to high magnitudes of tensile strain in monolayer may alter their cytoskeletal structure. Consequently, the resulting increased actin depolymerization in response to either high strain magnitudes or stress deprivation may actually be the stimulus for the up-regulation of interstitial collagenase reported in these studies. We hypothesize that cells subjected to high strains in monolayer culture significantly alter their cytoskeletal structure resulting in increased actin depolymerization, loss of cytoskeletal homeostatic tension, and increase in collagenase expression. 


\section{Materials and methods}

\section{Exercise Protocol}

Following Institutional Animal Care and Use approval and in accordance with the standards of the Muscle, Ligament, and Tendons Journal ${ }^{17}$, tendon cells from rat tail tendons were expanded from explant cultures and utilized in the study. All cells were from passage 2 to 5 . Rat-tail tendon cells were plated at 100,000 cells per well into 6 well collagen I coated BioFlex@ plates (Flexcell International Corp., Burlington, NC). Cells were allowed to adhere for 24 hours prior to a cyclic strain protocol and maintained in DMEM media supplemented with $10 \%$ FBS, $1 \%$ antibiotic/antimycotic solution, $0.2 \%$ gentamicin, and $7.5 \mathrm{~g} / \mathrm{L}$ ascorbate. Plates were paired into control and the following equibiaxial, cyclically tensile strained $(1 \mathrm{~Hz})$ groups ( $n=18$ wells per group): (1) $5 \%$ strain (2) $10 \%$ strain (3) $15 \%$ strain. The cells were cyclically strained using a Flexercell Strain Unit® with BioFlex Loading Stations $(25 \mathrm{~mm})$ (Flexcell International Corp., Burlington, NC) for 2 hours at $1 \mathrm{~Hz}$ using a sinusoidal strain pattern from $0 \%$ strain to the corresponding actual maximum strains of $4.75,9.5$, and $12 \%$.

\section{Actin staining}

The cells were fixed with $3.7 \%$ formaldehyde during the last 2 minutes of the cyclic strain protocol and then an additional 8 minutes with no strain for a total fixation time of 10 minutes. The fixative was removed and cells were washed with phosphate buffered saline (PBS). Cells were then incubated in $10 \%$ sucrose for 15 minutes after which the sucrose was removed and cells were rinsed with PBS. Cellular actin filaments were stained with rhodamine phalloidin (50 $\mathrm{U} / \mathrm{ml}$ ) solution (Invitrogen Corp., Carlsbad, CA) for 20 minutes. The stain was removed and cells were rinsed with PBS. The BioFlex@ membranes were cut from the flexcell plates and mounted cells-up on a glass slide. Vecta-mount with DAPI (Vector Laboratories, Burlingame, CA) was added to each membrane and then overlaid with a \#1 coverslip. Individual cells were photographed in the central portion of the membrane through the coverslip on a Zeiss Axiovert 200M microscope with a 40x objective (Zeiss International, Oberkochen, Germany).

\section{Actin Intensity}

The intensity of actin staining was used as an indicator of actin polymerization ${ }^{16}$. Each of the isolated cells photographed ( $n=420$ per group) were measured for actin staining intensity using Scion Image (Scion Corporation, Frederick, MD). For each image, the background optical density mode was subtracted from the cell's mean optical density to obtain the cell's actin intensity value. The cell's actin intensity values range from 0 to 255 with 0 corresponding to intense actin staining (white) and 255 corresponding to no actin staining (black). The average actin intensity for each of the three cyclically strained groups was then compared to its control group using a t-test with Bonferroni correction and significance set at $\mathrm{p}<0.0167$.

\section{Cell Conformation}

All of the photomicrographed cells in the paired $12 \%$ cyclic strain $(n=489)$ and control groups $(n=420)$ were additionally analyzed (Scion Image) to determine their cell conformation by measuring their length and width. For each cell, the conformation (ratio of cell length: width) was determined as the response variable. A cell conformation of 1 corresponded to a round cell while higher values corresponded to more elongate cells. Factors that could have affected cell conformation were treatment (cyclic strain vs control), plates (nested within treatments), and wells (nested within plates). The data were analyzed (SAS PROC MIXED) by means of a multi-nested ANOVA with $p$ values reported.

\section{MMP-13 Gene Expression Measurements}

Additional rat-tail tendon cells were plated and cultured in a similar manner as the previous exercise protocol. These cells were paired into control and cyclically loaded at $12 \%$ strain for 24 hours at $1 \mathrm{~Hz}$. After this loading protocol, rat-tail tendon cells were harvested immediately in RNAlater ${ }^{\mathrm{TM}}$ (Qiagen, Valencia, CA) to arrest all cellular metabolism. Total RNA from these and control (untreated) cells were then isolated using the RNeasy Plus Mini Kit (Qiagen). Approximately $400 \mathrm{ng}$ of RNA was then converted into cDNA using the Invitrogen SuperScript III Reverse Transcription system (Carlsbad, CA). Real Time Quantitative PCR was performed using the TaqMan ${ }^{\circledR}$ Gene Expression Assay from Applied Biosystems (ABI, Foster City, CA) in the ABI 7500 Fast System. Rat MMP-13 primers and $\operatorname{TaqMan}^{\circledR}$ probes (ID \# Rn01448197_m1) were obtained from the TaqMan ${ }^{\circledR}$ Gene Expression Assay database at ABI (http://allgenes.com). The endogenous control used for all QPCR experiments was Eukaryotic 18s rRNA (ID \# Hs99999901_s1). Results were analyzed using the Sequence Detection System software available from $\mathrm{ABI}$. Every treatment was repeated six times and results were statistically evaluated using a t-test with significance set at $\mathrm{p}<0.05$.

\section{Results}

There was no significant difference in the degree of actin staining intensity between the control group and cells strained at either 4.75 or $9.5 \%$ actual substrate strain (Tab. 1). However, cells strained at $12 \%$ actual substrate strain demonstrated a significant $(p<0.01)$ 
decrease in actin staining intensity when compared to control cells (Tab. 1). This would indicate a significant increase in actin depolymerization at this strain level. In addition, results of the cell conformation measurements and photomicrographs revealed a clear shift towards a more rounded cell shape in the $12 \%$ strain group (Figs. 1, 2).

The results of the cell conformation nested ANOVA are that there is significant variation among wells within plates $(P=0.0002)$ and significant variation

Table 1. Cellular Actin Staining Intensity Value (range 0-255 with 0 corresponding to intense staining and 255 corresponding to no staining).

\begin{tabular}{llll}
\hline & $\mathbf{5 \%}$ Strain & $\mathbf{1 0 \%}$ Strain & $\mathbf{1 5 \%}$ Strain \\
\hline Control & $154 \pm 6$ & $169 \pm 10$ & $155 \pm 19$ \\
Cyclic Strain & $163 \pm 13$ & $173 \pm 6$ & $191 \pm 13$ \\
p-value & 0.1560 & 0.3886 & 0.0009
\end{tabular}

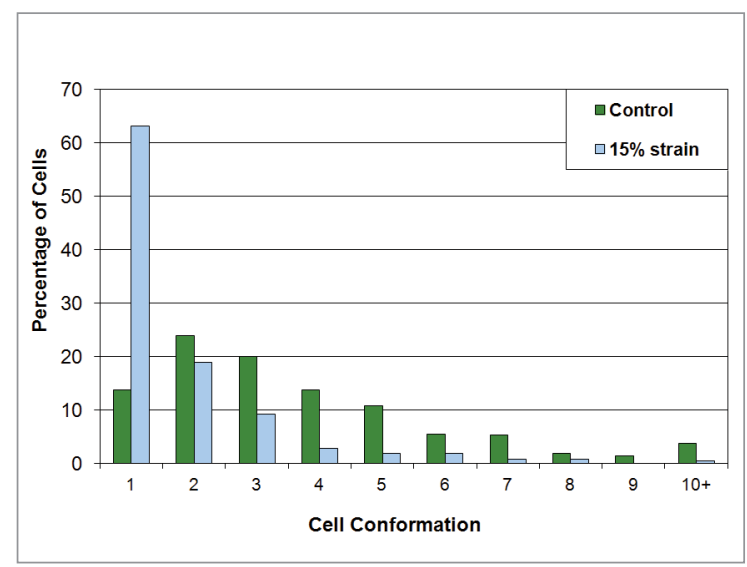

Figure 1. Histogram of percentage of cells within each cell conformation range for $15 \%$ strain as well as control cells. A cell conformation of 1 corresponds to a round cell while higher values correspond to more elongate cells. among plates $(\mathrm{P}<0.0001)$. However, regardless of well and plate variation, the mean conformation value for the control cells $(4.17+/-0.09)$ was significantly greater $(p<0.0001)$ than those cells undergoing $12 \%$ substrate strain $(2.17+/-0.10)$ (mean +/- SEM). Gene expression analysis revealed that although not statistically significant $(p=0.12)$, the average MMP-13 transcript levels in the cyclically strained cells $(38 \pm 21)$ were $81 \%$ higher than in the control cells $(21 \pm 2)$.

\section{Discussion}

The results of this study demonstrate that high (12\%) levels of in vitro, biaxial, substrate strain can significantly increase actin depolymerization, thereby altering the shape of tendon cells. However, exposure of tendon cells to lesser substrate strains ( $\leq 9.5 \%)$ caused no difference in their actin polymerization when compared to controls. This is in accord with a previous in vitro study in which $15 \%$ of applied cell strain to chondrocytes led to increased actin depolymerization ${ }^{16}$. It has been theorized that the increase in actin depolymerization seen in response to large substrate strains may actually decrease the cell modulus, enabling the cell to undergo large distortions without damage ${ }^{16,18}$. A previous monolayer study demonstrated that the cellular cytoskeleton, in response to transient stretch, fluidizes, or becomes depolymerized, with a prompt decrease in cell stiffness corresponding with the amplitude of the imposed stretch $^{19}$.

The actin cytoskeleton plays an important biomechanical function in determining cell-matrix interactions and regulation of the cellular response to mechanical stimuli ${ }^{18}$. In tendons and ligaments, the mechanobiological response of the cell is mediated, in large part, through the cytoskeleton, and alterations in the shape and structure of the cytoskeleton are known to influence a variety of gene expressions ${ }^{1,2}$. These alterations in cytoskeletal structure can also affect the mechanobiological response of the cells, as both changes in cell shape as well as the
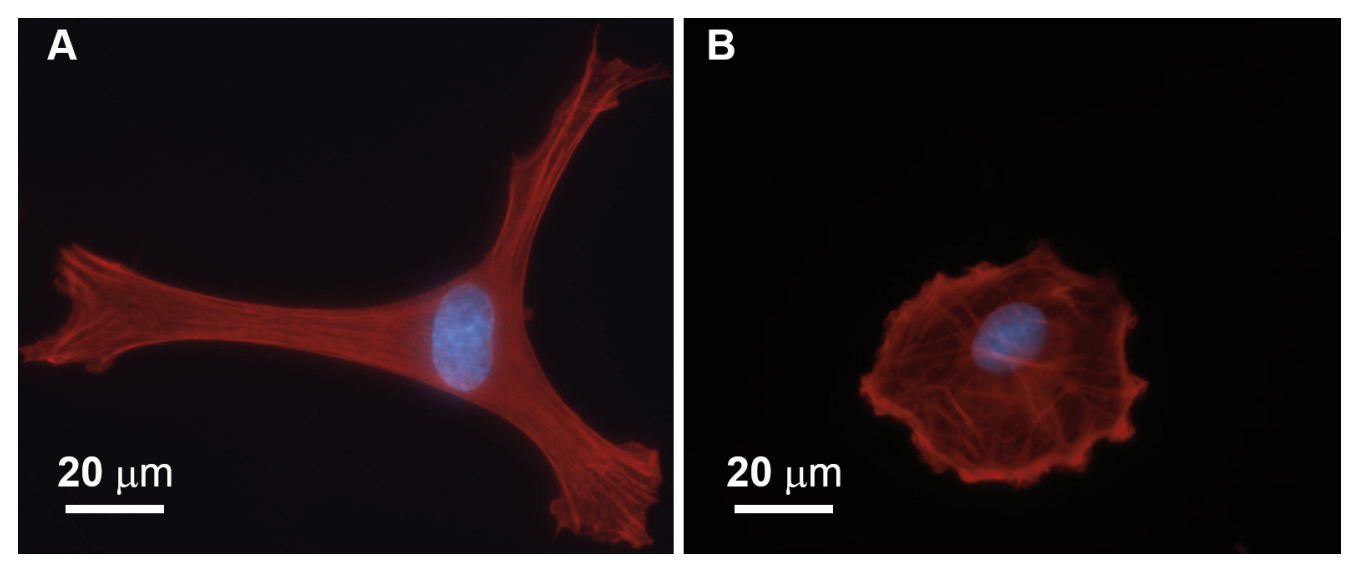

Figure 2. Representative photomicrographs of an elongate cell with intense fluorescence of actin stress fibers in the control group (A) and a round cell with less intense staining of the actin stress fibers in the $15 \%$ strain group (B). 
depolymerization of the actin cytoskeleton have been shown to influence collagenase gene expression $^{2,11,15}$. Tendon cells are also capable of actively contracting their extracellular matrix through an alpha smooth muscle actin based mechanism ${ }^{12,20}$. This contraction can re-establish the cytoskeletal tensional homeostasis of these tendon cells in situ by recalibrating their mechanobiological set point with respect to collagenase protein synthesis 20 .

While not significantly different, the results of the current study demonstrate an increase in collagenase expression ( $81 \%$ ) in cells undergoing $12 \%$ biaxial cyclic strain compared to controls. Previous in vitro studies have shown a threshold effect with similar small increases in collagenase with high strains and a minimal change in collagenase at lower strains ${ }^{6,8}$. In one such study, applied equibiaxial cyclic tensile strains of 15 and $10 \%$ resulted in a slight up-regulation $(58 \%)$ or down regulation $(30 \%)$ of collagenase content respectively ${ }^{6}$. Another in vitro study showed that uniaxial tensile cyclic strains of $8 \%$ increased the expression of collagenase by $145 \%$, while $4 \%$ strain increased it by only $20 \% 8$. This threshold effect seen with the amount of applied strain and collagenase expression in previous studies coincides with the threshold level of actin depolymerization seen in the current study. While the cells in the current study were exposed to cyclic equibiaxial strain (Flexcell International Corp., Burlington, NC), it was recently demonstrated experimentally and computationally that the strain level is not uniform across the well21. Cells in the central portion of the well were shown to experience strains of up to $2 \%$ less than the mean value $^{21}$. This heterogeneous strain field may explain the highly variable collagenase expression that resulted in a non-statistically significant comparison to controls in the current study.

Abnormal tendon cell stimulation has been suggested to play a role in the etiology of tendinopathy ${ }^{3,4}$. Several investigators have suggested that it is the mechanobiological response of the cells to repetitive overloading that initiates the degenerative cascade that leads to tendinopathy ${ }^{6-8}$. However, the use of high magnitude cyclic strain to elicit a relatively small catabolic gene expression in tendon and ligament cells cultured on artificial substrates may not be clinically relevant ${ }^{5-7}$. Although tendons may naturally undergo strains greater than $12 \%$, these strains have been shown to lead to isolated collagen fibril damage ${ }^{13,22}$. In addition, tendon cell strain in situ has been shown to be significantly less than whole tendon strain ${ }^{3}$. Therefore, it is unlikely that such high levels of repetitive tendon cell strain could be reached and maintained in vivo without significant damage occurring within the extracellular matrix of the tendon.

In experimental studies, tendons have been loaded to induce isolated fibrillar damage, and these damaged fibrils have been shown to relax and lose their ability to transmit load ${ }^{13,23}$. This inability of the damaged fibrils to transmit load prevents them from maintaining a homeostatic mechanobiological stimulus to those cells associated with the damaged fibrils ${ }^{13}$. Consequently, alterations of cell-matrix interaction, secondary to isolated fibrillar damage, could result in a mechanobiological under-stimulation of tendon cells. This loss of homeostatic tension within the cytoskeleton due to loss of extracellular matrix tension has been documented in rat tail tendons, and results in the alteration of cell nuclear shape ${ }^{10}$ and a significant increase in collagenase mRNA expression and protein synthesis ${ }^{11,13}$. Therefore, the exposure of cells to either high strain in vitro or loss of strain in situ results in cytoskeletal depolymerization, loss of cytoskeletal tensional homeostasis, and alteration of cell shape.

Although the exposure of cells to either strain or loss of strain may appear to result in initially similar outcomes, the difference in the response could be due based on temporal reasons. Cells exposed to high strains, that are non-damaging to the matrix, will recover their actin cytoskeletal stiffness within $200 \mathrm{sec}-$ ond after loading ${ }^{19}$. Cells that are exposed to the loss of matrix tension however, lose their tensional homeostasis until the matrix tension can be restored which may take days ${ }^{20}$, with loss of substrate elasticity inducing a higher level of collagenase gene expres$\operatorname{sion}^{24}$. The restoration of tensional homeostasis in lax tendons can occur through an actin-mediated cellular contraction of the extracellular matrix which may be an important mechanism in the recovery of matrix tension in tendons 25 .

The results of the current study suggest that the upregulation of collagenase associated with the application of high magnitude $(>12 \%)$ tensile strains ${ }^{6}$ may actually be a result of cytoskeletal depolymerization, loss of cytoskeletal tensional homeostasis, and alteration of cell shape. Because it is unlikely that cells in situ are ever exposed to such high tensile strains ${ }^{3}$, the mechanism(s) responsible for the up-regulation of collagenase seen in repetitive use injuries ${ }^{4}$ may not, in fact, be due to over-stimulation of cells but rather an understimulation of cells and subsequent loss of cytoskeletal tensional homeostasis secondary to alterations in the extracellular matrix ability to transfer loads ${ }^{23}$.

\section{Conflict of Interest}

No benefits in any form have been or will be received from a commercial party related directly or indirectly to the subject of this manuscript.

\section{References}

1. Chicurel ME, Chen CS, Ingber DE. Cellular control lies in the balance of forces. Curr Opin Cell Biol. 1998;0:232-239.

2. Unemori EN, Werb Z. Reorganization of polymerized actin: a possible trigger for induction of procollagenase in fibroblasts cultured in and on collagen gels. J Cell Biol. 1986;103:10211031.

3. Arnoczky SP, Lavagnino M, Whallon JH, Hoonjan A. In situ cell nucleus deformation in tendons under tensile load; a morpho- 
logical analysis using confocal laser microscopy. J Orthop Res. 2002;20:29-35.

4. Jozsa LG, Kannus P. Human Tendons: Anatomy, Physiology, and Pathology. Champaign, IL; Human Kinetics. 1997.

5. Almekinders LC, Banes AJ, Ballenger CA. Effects of repetitive motion on human fibroblasts. Med Sci Sports Exerc. 1993;25: 603-607.

6. Bhargava M, Attia E, Hannafin JA. The effect of cyclic tensile strain on MMPs, collagen and casein degrading activities of fibroblasts isolated from anterior cruciate and medical collateral ligaments. Trans Ortho Res Soc. 2004;29:110.

7. Wang JH, Jia F, Yang G, et al. Cyclic mechanical stretching of human tendon fibroblasts increases the production of prostaglandin E2 and levels of cyclooxygenase expression: a novel in vitro model study. Connect Tissue Res. 2003;44:128-133.

8. Yang G, Im HJ, Wang JH. Repetitive mechanical stretching modulates IL-1beta induced COX-2, MMP-1 expression, and PGE2 production in human patellar tendon fibroblasts. Gene. 2005;363:166-172.

9. Del Buono A, Oliva F, Osti L, Maffulli N. Metalloproteases and tendinopathy. Muscles Ligaments Tendons J. 2013;3:51-57.

10. Arnoczky SP, Lavagnino M, Egerbacher M, Caballero O, Gardner K, Shender MA. Loss of homeostatic strain alters mechanostat "set point" of tendon cells in vitro. Clin Orthop Relat Res. 2008;466:1583-1591.

11. Arnoczky SP, Tian T, Lavagnino M, Gardner K. Ex vivo static tensile loading inhibits MMP-1 expression in rat tail tendon cells through a cytoskeletally based mechanotransduction mechanism. J Orthop Res. 2004;22:328-333.

12. Lavagnino M, Arnoczky SP. In vitro alterations in cytoskeletal tensional homeostasis control gene expression in tendon cells. J Orthop Res. 2005;23:1211-1218.

13. Lavagnino M, Arnoczky SP, Egerbacher M, Gardner KL, Burns ME. Isolated fibrillar damage in tendons stimulates local collagenase mRNA expression and protein synthesis. J Biomech. 2006;39:2355-2362.

14. Majima T, Marchuk LL, Shrive NG, Frank CB, Hart DA. In-vitro cyclic tensile loading of an immobilized and mobilized ligament autograft selectively inhibits mRNA levels for collagenase (MMP-1). J Orthop Sci. 2000;5:503-510.
15. Lambert CA, Colige AC, Munaut C, Lapière CM, Nusgens BV Distinct pathways in the over-expression of matrix metalloproteinases in human fibroblasts by relaxation of mechanical tension. Matrix Biol. 2001;20:397-408.

16. Campbell JJ, Blain EJ, Chowdhury TT, Knight MM. Loading alters actin dynamics and up-regulates cofilin gene expression in chondrocytes. Biochem Biophys Res Commun. 2007;361: 329-34.

17. Padulo J, Oliva F, Frizziero A, Maffulli N. Muscles, Ligaments and Tendons Journal. Basic principles and recommendations in clinical and field science. MLTJ. 2013;4:250-252.

18. Trickey WR, Vail TP, Guilak F. The role of the cytoskeleton in the viscoelastic properties of human articular chondrocytes. $J$ Orthop Res. 2004;22:131-139.

19. Trepat X, Deng L, An SS, et al. Universal physical responses to stretch in the living cell. Nature. 2007;447:592-595.

20. Gardner K, Lavagnino M, Egerbacher M, Arnoczky SP. Reestablishment of cytoskeletal tensional homeostasis in lax tendons occurs through an actin-mediated cellular contraction of the extracellular matrix. J Orthop Res. 2012;30:16951701.

21. Olesen CG, Pennisi CP, de Zee M, Zachar V, Rasmussen J. Elliptical posts allow for detailed control of non-equibiaxial straining of cell cultures. J Tissue Viability. 2013;22:52-56.

22. Lavagnino M, Arnoczky SP, Elvin N, Dodds J. Patellar tendon strain is increased at the site of the jumper's knee lesion during knee flexion and tendon loading: results and cadaveric testing of a computational model. Am J Sports Med. 2008;36:21102118.

23. Knörzer E, Folkhard W, Geercken W, et al. New aspects of the etiology of tendon rupture. An analysis of time-resolved dynamic-mechanical measurements using synchrotron radiation. Arch Orthop Trauma Surg. 1986;105:113-120.

24. Maeda E, Sugimoto M, Ohashi T. Cytoskeletal tension modulates MMP-1 gene expression from tenocytes on micropillar substrates. J Biomech. 2013;46:991-997.

25. Lavagnino M, Bedi A, Walsh CP, Sibilsky Enselman ER, Sheibani-Rad S, Arnoczky SP. Tendon contraction after cyclic elongation is an age-dependent phenomenon: in vitro and in vivo comparisons. Am J Sports Med. 2014;42:1471-1477. 